\title{
Heterocedasticidade entre fileiras e colheitas de caracteres produtivos de tomate cereja e estimativa do tamanho de parcela
}

\author{
Alessandro DC Lúcio; Bruno G Sari; Rafael V Pezzini; Victor Liberalesso; Fabrício Delatorre; Maurício Faé \\ Universidade Federal de Santa Maria (UFSM), Santa Maria-RS, Brasil; adlucio@ufsm.br; brunosari@hotmail.com; rvpezzini@hotmail. \\ com; victor@drakkarsolos.com.br; fbdelatorre@gmail.com; mauricio.fae@hotmail.com
}

\section{RESUMO}

O presente estudo teve por objetivo avaliar a heterocedasticidade entre as fileiras de cultivo e entre as colheitas em tomate cereja cultivado em ambiente protegido e determinar o tamanho ótimo de parcela para avaliar as características produtivas da cultura. Para isso instalou-se dois ensaios de uniformidade em estufas plásticas, cada uma contendo oito fileiras espaçadas $1 \mathrm{~m}$ entre si. Utilizou-se o híbrido Lili, e as mudas foram espaçadas em $0,5 \mathrm{~m}$. Foram realizadas três colheitas e as análises foram realizadas com base nas colheitas individuais e agrupadas. A homocedasticidade entre as fileiras de cultivo e entre as colheitas para cada fileira de cultivo foi obtida pelo teste de Bartlett. O tamanho de parcela (em número de plantas) foi calculado para cada fileira e colheita (individual ou agrupada). Observou-se heterocedasticidade entre as fileiras de cultivo para pelo menos um caractere em todas as colheitas (individuais ou agrupadas). Ocorreu heterocedasticidade entre as colheitas dentro de cada fileira de cultivo para todas as variáveis. Estas duas características justificam a necessidade de se estimar o tamanho ótimo de parcela para cada situação. O tamanho de parcela (em número de plantas) foi diferenciado entre as fileiras e as colheitas, sendo esta estimativa menor quando as três colheitas são agrupadas. É recomendado o agrupamento das colheitas e a adoção de parcelas contendo sete plantas de tomate, independente da fileira de cultivo.

Palavras-chave: Lycopersicon esculentum, olericultura, ambiente protegido, precisão experimental.

\begin{abstract}
Heteroscedasticity between rows and harvests of cherry tomato yield characters and estimate of the plot size

We studied the heteroscedasticity between rows and harvests of cherry tomato cultivated under protected environment and we determined the optimum plot size to evaluate yield characteristics. Two uniformity experiments were set under greenhouses, each containing eight crop rows spaced $1 \mathrm{~m}$. The hybrid Lili was used, and the plantlets were spaced $0.5 \mathrm{~m}$. Three harvests were carried out, and the data analysis was carried out based on the individual and combined harvests. The homoscedasticity among the rows and the harvests was obtained by Bartlett test. The plot size (in number of plants) was calculated for each row and each harvest (individual or combined). Heteroscedasticity among rows was observed for at least one character in all harvests (individual or combined). Heteroscedasticity was recorded among harvests in each row for all characters. These two observations justify the need to estimate the optimum plot size for each situation. The plot size (in number of plants) was different between lines and crops, the plot size estimate being smaller when the three harvests are grouped. The grouping of harvests and the use of plots containing seven tomato plants are recommended, regardless the crop row.
\end{abstract}

Keywords: Lycopersicon esculentum, horticulture, protected environment, experimental precision.

(Recebido para publicação em 17 de março de 2015; aceito em 14 de janeiro de 2016) (Received on March 17, 2015; accepted on January 14, 2016)

$\mathrm{O}$ cultivo do tomate (Lycopersicon esculentum) é uma importante atividade agrícola no Brasil. Na safra 2013 o tomateiro foi cultivado em mais de 60 mil hectares onde foram produzidos 3,9 milhões de toneladas do fruto (IBGE, 2014). Entre os diversos tipos de tomate, o cereja destaca-se por sua utilização como adorno em pratos ou aperitivo (Gusmão et al., 2000) e por apresentar valores mais elevados no mercado em comparação às outras variedades de tomate.
O cultivo de hortaliças, e entre elas pode-se citar o tomate, é realizado predominantemente a campo. Porém, o cultivo em ambiente protegido apresenta vantagens como a maior precocidade na produção, o melhor controle de pragas e doenças, a economia e melhor aproveitamento da água de irrigação (Cermeño, 1990) e a possibilidade de produção fora da época preferencial em locais onde há limitação devido a condições ambientais inadequadas (Lorentz et al., 2004). Devido a isso, o cultivo do tomateiro em ambientes protegidos tem se expandido principalmente na região sudeste, uma das maiores produtoras do Brasil (Gusmão et al., 2006).

O cultivo do tomate cereja em ambiente protegido demanda estudos que testem formas de manejo que maximizem a sua produção. Ao realizar um experimento, o pesquisador espera que a variabilidade entre as parcelas seja consequência do efeito dos seus tratamentos (Lúcio et al., 2008), e para que isso ocorra, o erro experimental deve 
ser minimizado. $\mathrm{O}$ dimensionamento adequado ao número de plantas por parcela, tamanho da amostra, número de repetições, delineamento experimental, entre outros, são formas de minimizar o erro experimental (Storck et al., 2006). As formas de melhoria da precisão experimental descritas acima são influenciadas pela variabilidade inerente ao experimento (Steel et al., 1997; Storck et al., 2006), a qual deve ser estudada para que o planejamento experimental seja adequado.

Em experimentos com olerícolas de colheitas múltiplas cultivadas em ambiente protegido, a variabilidade dos dados pode estar relacionada a diversos fatores: a) variabilidade entre colheitas, devido a maturação desuniforme dos frutos; b) variabilidade entre as fileiras de cultivo, devido a proximidade com a lateral das estufas; c) o manejo intensivo (controle de pragas e doenças, adubação, eliminação de brotos laterais, entre outros) realizado nas estufas plásticas (Cargnelutti Filho et al., 2004; Lorentz et al., 2005; Lúcio et al. 2008, 2010). Diversos autores, trabalhando em ambiente protegido com olerícolas de colheitas múltiplas, relatam que os fatores citados acima alteram as estimativas dos tamanhos de amostra, tamanho e forma de parcela, delineamento experimental e número de colheitas suficientes para discriminar os tratamentos estudados (Cargnelutti Filho et al., 2004; Lúcio et al., 2004, 2006, 2008, 2010; Lorentz et al., 2005; Feijó et al., 2008).

Deste modo, há necessidade de se minimizar as fontes de variabilidade existentes em culturas de produção escalonada (colheitas múltiplas) cultivadas em ambiente protegido. Trabalhar com tamanhos de parcela que contenham um número adequado de plantas ou agrupar colheitas são formas de reduzir a variabilidade existente neste tipo de experimento. Resultados similares já foram encontrados para diversas olerícolas de múltiplas colheitas, tais como o pimentão (Lúcio et al., 2004; Lorentz et al., 2005), a abobrinha italiana (Lúcio et al., 2008; Carpes et al., 2010), o tomate tipo salada (Lúcio et al., 2010) e o feijão-vagem (Santos et al., 2012), porém não foram encontrados trabalhos similares com tomate do tipo cereja.
Diante do exposto, esse trabalho teve por objetivo estudar a heterocedasticidade entre as fileiras de cultivo e entre as colheitas de tomate cereja cultivado em ambiente protegido e determinar o tamanho ótimo de parcela para avaliar seus caracteres produtivos.

\section{MATERIAL E MÉTODOS}

Dois ensaios de uniformidade foram realizados em estufas plásticas do tipo arco pampeano, ambas orientadas no sentido norte-sul e com dimensões distintas: 1) estufa pequena, com pé direito de $3,00 \mathrm{~m}$ e 4,00 $\mathrm{m}$ na parte central, com $20,00 \mathrm{~m}$ de comprimento e $10,00 \mathrm{~m}$ de largura; 2) estufa grande, com pé direito de 4,50 $\mathrm{m}$ e 5,50 $\mathrm{m}$ na parte central, com $25,00 \mathrm{~m}$ de comprimento e $10,00 \mathrm{~m}$ de largura. Ambas as estufas estavam cobertas com filme de polietileno de baixa densidade (PEBD), com espessura de 150 micras e aditivo anti-UV.

Os experimentos foram realizados com o híbrido de tomate do tipo cereja Lili na estação primavera/verão de 2014, no Departamento de Fitotecnia da Universidade Federal de Santa Maria $\left(29^{\circ} 43^{\circ} \mathrm{S}, 53^{\circ} 43^{\circ} \mathrm{O}\right.$, altitude 95 m). O clima é classificado como Cfa (subtropical úmido sem estação seca definida e com verões quentes) e o solo é classificado como Argissolo Vermelho distrófico arênico (Embrapa, 2006).

No mês anterior à instalação do experimento o $\mathrm{pH}$ do solo foi elevado a 6,5 e os níveis de fósforo a $300 \mathrm{mg} /$ $\mathrm{dm}^{3} \mathrm{em}$ ambas as estufas. O calcário e o fósforo utilizados na correção do solo foram incorporados com uma enxada rotativa, sendo que posteriormente foram erguidos camalhões com aproximadamente $20 \mathrm{~cm}$ de altura e 30 $\mathrm{cm}$ de largura, cobertos com faixas de mulching de filme opaco de PEBD de cor preta. Em cada estufa foram erguidos oito camalhões espaçados em $1 \mathrm{~m}$ entre si, e a irrigação das plantas foi realizada por gotejamento.

O transplante das mudas foi realizado no dia 26 de outubro em ambas as estufas. Conforme recomendação da empresa detentora do híbrido, as mudas foram transplantadas quando emitiram a primeira florada. O espaçamento entre mudas utilizado foi de $50 \mathrm{~cm}$ e a adubação foi realizada com $150 \mathrm{~kg} / \mathrm{ha}$ de N, $250 \mathrm{~kg} / \mathrm{ha}$ de P e $125 \mathrm{~kg} / \mathrm{ha}$ de K em ambas as estufas. Foram realizadas duas adubações de cobertura com $30 \mathrm{~kg} /$ ha de N, $15 \mathrm{~kg} / \mathrm{ha}$ de $\mathrm{P}$ e $30 \mathrm{~kg} / \mathrm{ha}$ de $\mathrm{K}$ em um intervalo de 23 dias. A adubação de base e cobertura foi realizada baseada na análise química do solo e de acordo com a recomendação da Rede Oficial de Laboratórios de Análise de Solo e de Tecido Vegetal dos Estados do Rio Grande do Sul e de Santa Catarina (ROLAS, 2004).

As plantas de tomate foram tutoradas com duas hastes em ambas as estufas, sendo eliminados todos os brotos laterais. A cada 14 dias foram realizadas pulverizações foliares de cálcio e boro com o objetivo de evitar o "fundo preto" nos frutos. Também foram realizadas aplicações de fungicidas e inseticidas a cada 14 dias com o objetivo de controlar preventivamente pragas e doenças.

Cada planta foi considerada uma unidade experimental básica (UB), e devido ao comprimento diferenciado, o número de UB's nas fileiras de cultivo foi distintos entre as estufas. Todos os cachos de todas as UB's foram colhidos, sendo considerado o cacho apto para colheita aquele em que todos os frutos apresentavam mudança de coloração de verde para avermelhado. Todos os frutos de todos os cachos foram medidos, com auxílio de um paquímetro e pesados com auxílio de uma balança de precisão $0,01 \mathrm{~g}$.

As variáveis avaliadas nos cachos e frutos colhidos foram: comprimento médio de fruto por planta (CMF), que corresponde à medida transversal do fruto, em centímetros; largura média de fruto por planta (LMF), que corresponde à medida longitudinal do fruto, em centímetros; peso médio de fruto por planta (PMF) em gramas; número de cachos por planta $(\mathrm{NCP})$; número de frutos por cacho (NFC); número de frutos por planta (NFP); peso total de frutos por planta (PTF) em gramas.

Foram realizadas três colheitas, sendo que elas foram analisadas individualmente $\left(1^{\mathrm{a}} ; 2^{\mathrm{a}}\right.$ e $\left.3^{\mathrm{a}}\right)$ e agrupadas $\left(1^{\mathrm{a}}+2^{\mathrm{a}} ; 2^{\mathrm{a}}+3^{\mathrm{a}} ; 1^{\mathrm{a}}+2^{\mathrm{a}}+3^{\mathrm{a}}\right)$. Para todas as variáveis foi testada a homogeneidade das variâncias entre as fileiras de cul- 
tivos para cada colheita (individual e agrupada) e entre as colheitas (individuais e agrupadas) para cada fileira de cultivo. Para estas análises utilizou-se o teste de Bartlett (Steel et al., 1997), já que os dados aderiram-se à distribuição normal de acordo com o teste prévio de Shapiro-Wilk.

Posteriormente, para cada uma das colheitas (individuais e agrupadas) e em cada fileira de cultivo, foram estimadas a variância $\left(\mathrm{s}^{2}\right)$, a média $(\bar{x})$, os coeficiente de autocorrelação espacial de primeira ordem $(\hat{\rho})$, pela expressão

$$
\hat{\rho}=\frac{\sum_{i=1}^{r c}\left(\hat{\varepsilon}_{i}-\bar{\varepsilon}\right)\left(\hat{\varepsilon}_{i-1}\right)}{\sum_{i=1}^{r c}\left(\hat{\varepsilon}_{i}-\bar{\varepsilon}\right)^{2}}
$$

em que $\hat{\varepsilon}$ é o erro experimental associado a cada observação, e o tamanho ótimo de parcela ( $\left.\hat{X}_{O}\right)$ pela expressão:

$$
\hat{X}_{O}=\frac{10 \sqrt[3]{2\left(1-\hat{\rho}^{2}\right) s^{2} \bar{x}}}{\bar{x}}
$$

(Paranaíba et al., 2009).

A relação existente entre os valores estimados das estatísticas autocorrelação espacial de primeira ordem $(\hat{\rho})$, a variância $\left(\mathrm{s}^{2}\right)$, a média $(\bar{x})$, o tamanho ótimo de parcela ( $\hat{X}_{o}$ ) foi testada pelo coeficiente de correlação de Pearson, e a sua significância foi testada pelo teste $t$. Todas as análises foram realizadas com auxílio do software R versão 2.15.1 (R Development Core Team, 2014) e do aplicativo Office Excel, adotando 5\% de significância.

\section{RESULTADOS E DISCUSSÃO}

Foram realizadas três colheitas em ambas as estufas, com um período médio entre as colheitas de 13,5 dias. $\mathrm{O}$ número de colheitas foi menor e o período entre as colheitas maior quando comparado ao tomate do tipo salada. Lucio et al. (2012) realizaram 12 colheitas com um período entre colheitas de 3,5 dias em trabalhos com este tipo de tomate. $\mathrm{O}$ número e o período entre colheitas deste experimento também difere de resultados obtidos por Gusmão et al. (2006), Azevedo et al. (2010) e Rocha et al. (2010) em experimentos com tomate cereja. Os trabalhos citados relatam períodos entre colheitas de 3,5 e 7 dias, totalizando até 10 colheitas durante o ciclo.

$\mathrm{O}$ número reduzido de colheitas neste trabalho pode estar relacionado ao fato de os cachos terem sido colhidos inteiros, o que pode resultar em um menor número e frequência de colheitas, pois todos os frutos do cacho deviam estar aptos a ser colhidos. Além disso, a mensuração de todas as variáveis em laboratório demandava tempo, uma vez que no total foram avaliados 72.173 frutos, o que não permitiu uma maior frequência de colheitas nas estufas.

Ressalta-se, também, que não é nosso objetivo determinar o número mínimo de colheitas necessário para discriminar tratamentos ou genótipos em experimentos com tomate tipo cereja, sendo este um importante estudo a ser realizado futuramente. Mesmo com poucas colheitas, as estimativas do tamanho de parcela deste experimento podem ser amplamente utilizadas, principalmente aquelas que representam menos de $1 / 3$ da produção total e foram realizadas no início e fim do ciclo (Tabela 1). A estimativa do tamanho de parcelas nestes casos leva em conta a elevada variabilidade nestas colheitas, decorrente da ausência de plantas com frutos aptos a serem colhidos devido à maturação desuniforme dos frutos nas plantas de tomate (Cargnelutti Filho et al., 2004; Lúcio et al., 2010).

Em ambas as estufas houve hetero-

Tabela 1. $P$-valor do teste de Bartlett para a homogeneidade das variâncias entre fileiras de cultivo para colheitas individuais e agrupadas para as variáveis comprimento médio de fruto (CMF), largura média de fruto (LMF), peso médio de fruto (PMF), número de cachos por planta (NCP), número de frutos por cacho (NFC), número de frutos por planta (NFP) e peso total de fruto $(\mathrm{PTF})\{P$-value of Bartlett's test for the homogeneity of variances among the crop rows for each individual and harvests, grouped, from the variables average length of fruit (CMF), average width of fruit (LMF), average weight of fruit (PMF), number of bunches per plant (NCP), number of fruits per bunch (NFC), number of fruits per plant (NFP) and total weight of fruits (PTF) \}. Santa Maria, UFSM, 2014.

\begin{tabular}{lcccccc}
\hline \multirow{2}{*}{ Variáveis } & \multicolumn{7}{c}{ Estufa pequena } \\
\cline { 2 - 7 } & C1 $^{\mathbf{1}}$ & $\mathbf{C 2}$ & $\mathbf{C 3}$ & $\mathbf{C 1 + C 2}$ & $\mathbf{C 2 + C 3}$ & Todas \\
\hline CMF & 0,000 & 0,000 & 0,000 & 0,000 & 0,000 & 0,543 \\
LMF & 0,000 & 0,000 & 0,000 & 0,000 & 0,000 & 0,000 \\
PMF & 0,000 & 0,000 & 0,000 & 0,000 & 0,000 & 0,086 \\
NCP & 0,177 & 0,328 & 0,214 & 0,272 & 0,516 & 0,302 \\
NFC & 0,002 & 0,001 & 0,050 & 0,035 & 0,042 & 0,011 \\
NFP & 0,455 & 0,014 & 0,000 & 0,305 & 0,040 & 0,445 \\
PTF & 0,566 & 0,019 & 0,000 & 0,441 & 0,041 & 0,535 \\
\% de frutos ${ }^{2}$ & 52,32 & 35,85 & 11,83 & 88,17 & 47,68 & 100 \\
\hline & & \multicolumn{5}{c}{ Estufa grande } \\
\hline CMF & 0,003 & 0,097 & 0,002 & 0,000 & 0,000 & 0,000 \\
LMF & 0,005 & 0,115 & 0,001 & 0,000 & 0,000 & 0,000 \\
PMF & 0,019 & 0,230 & 0,005 & 0,000 & 0,002 & 0,000 \\
NCP & 0,077 & 0,000 & 0,185 & 0,001 & 0,062 & 0,079 \\
NFC & 0,041 & 0,267 & 0,000 & 0,000 & 0,142 & 0,055 \\
NFP & 0,256 & 0,030 & 0,000 & 0,054 & 0,112 & 0,244 \\
PTF & 0,285 & 0,053 & 0,028 & 0,068 & 0,130 & 0,284 \\
\% de frutos & 14,20 & 60,98 & 24,82 & 75,18 & 85,80 & 100 \\
\hline
\end{tabular}

${ }^{1} \mathrm{C} 1=$ primeira colheita (first harvest); $\mathrm{C} 2=$ segunda colheita (second harvest); $\mathrm{C} 3=$ terceira colheita (third harvest); $\mathrm{C} 1+\mathrm{C} 2=$ primeira e segunda colheitas agrupadas (first and second harvests, grouped); $\mathrm{C} 2+\mathrm{C} 3=$ segunda e terceira colheitas agrupadas (second and third harvests, grouped); Todas $=$ todas as colheitas agrupadas (all harvests, grouped) ${ }^{2}$ Porcentagem do total de frutos colhidos em cada colheita individual ou agrupada (percentage of total harvested fruits in each individual or harvests, grouped). 
Tabela 2. $P$-valor do teste de Bartlett para a homogeneidade das variâncias entre colheitas individuais e agrupadas em cada fileira de cultivo para as variáveis comprimento médio de fruto (CMF), largura média de fruto (LMF), peso médio de fruto (PMF), número de cachos por planta (NCP), número de frutos por cacho (NFC), número de frutos por planta (NFP) e peso total de fruto $(\mathrm{PTF})\{P$-value of Bartlett's test for the variances homogeneity among the individual and harvests, grouped in each crop row for the variables average length of fruit (CMF), average width of fruit (LMF), average weight of fruit (PMF), number of bunches per plant (NCP), number of fruits per bunch (NFC), number of fruits per plant (NFP) and total weight of fruit (PTF) \}. Santa Maria, UFSM, 2014.

\begin{tabular}{lccccccc}
\hline \multirow{2}{*}{ Variáveis } & \multicolumn{7}{c}{ Estufa pequena } \\
\cline { 2 - 8 } & CMF & LMF & PMF & NCP & NFC & NFP & PTF \\
\hline L1 & 0,000 & 0,000 & 0,000 & 0,000 & 0,000 & 0,000 & 0,000 \\
L2 & 0,000 & 0,000 & 0,000 & 0,000 & 0,000 & 0,000 & 0,000 \\
L3 & 0,000 & 0,000 & 0,000 & 0,000 & 0,000 & 0,000 & 0,000 \\
L4 & 0,000 & 0,000 & 0,000 & 0,000 & 0,000 & 0,000 & 0,000 \\
L5 & 0,000 & 0,000 & 0,000 & 0,025 & 0,000 & 0,001 & 0,000 \\
L6 & 0,000 & 0,000 & 0,000 & 0,000 & 0,000 & 0,000 & 0,000 \\
L7 & 0,000 & 0,000 & 0,000 & 0,000 & 0,000 & 0,000 & 0,000 \\
L8 & 0,000 & 0,000 & 0,000 & 0,000 & 0,000 & 0,000 & 0,000 \\
\hline \multicolumn{7}{c}{ Estufa grande } \\
\hline L1 & 0,000 & 0,000 & 0,000 & 0,000 & 0,000 & 0,000 & 0,000 \\
L2 & 0,000 & 0,000 & 0,000 & 0,000 & 0,000 & 0,000 & 0,000 \\
L3 & 0,000 & 0,000 & 0,000 & 0,000 & 0,000 & 0,000 & 0,000 \\
L4 & 0,000 & 0,000 & 0,000 & 0,000 & 0,000 & 0,000 & 0,000 \\
L5 & 0,000 & 0,000 & 0,000 & 0,000 & 0,000 & 0,000 & 0,000 \\
L6 & 0,000 & 0,000 & 0,000 & 0,000 & 0,000 & 0,000 & 0,000 \\
L7 & 0,000 & 0,000 & 0,000 & 0,000 & 0,000 & 0,000 & 0,000 \\
L8 & 0,000 & 0,000 & 0,000 & 0,000 & 0,000 & 0,000 & 0,000 \\
\hline
\end{tabular}

${ }^{1} \mathrm{~L} 1$ a L8= fileiras 1 a 8 (rows 1 to 8 ).

cedasticidade entre as fileiras de cultivo para pelo menos uma variável estudada, independente ou não do agrupamento das colheitas. As fileiras são homocedásticas para um maior número de variáveis quando as três colheitas são agrupadas $\left(1^{\mathrm{a}}+2^{\mathrm{a}}+3^{\mathrm{a}}\right)$ em comparação as colheitas individuais e agrupadas duas a duas na estufa pequena. Já na estufa grande, este comportamento não é observado, uma vez que a $2^{\mathrm{a}}$ colheita apresentou homocedasticidade entre as fileiras de cultivo para um maior número de variáveis (Tabela 1). Este resultado esta relacionado ao fato de que nesta colheita todas as plantas de tomate estão produzindo frutos, reduzindo o número de zeros no banco de dados. Além disso, não há impacto da variabilidade decorrente do início e término de maturação desuniforme dos frutos nesta colheita, como é comum em colheitas realizadas no início e fim do ciclo. Cargnelutti Filho et al. (2004) também verificaram que a variabilidade da produção de frutos de tomate salada em colheitas intermediárias é menor do que em colheitas realizadas no início e término do ciclo.

A heterocedasticidade entre fileiras de cultivo em estufas plásticas já havia sido relatada para as culturas do pimentão por Lúcio et al. (2003) e Lorentz et al. (2005) e da abobrinha italiana por Lúcio et al. (2008). Feijó et al. (2005) relatam que a heterogeneidade entre as fileiras de cultivo podem estar relacionadas à proximidade com as laterais da estufa. Além da proximidade com as laterais da estufa, Lúcio et al. (2008) relatam que a heterogeneidade entre as fileiras também pode estar relacionada pelas condições climáticas e pelo tipo de manejo. Todos estes trabalhos corroboram com os resultados encontrados neste estudo, porém cabe ressaltar que a maioria deles trabalhou com a fitomassa fresca de frutos, uma vez que trabalhos que abrangem vários caracteres produtivos são pouco encontrados na literatura.

Observou-se também heterocedasticidade entre as colheitas (individuais e agrupadas) para uma mesma fileira de cultivo para todas as variáveis em ambas as estufas, uma vez que todos os $p$-valores foram menores que 0,05 (Tabela 2). As variáveis estudadas apresentam diferenças que interferem na explicação deste resultado. No caso das variáveis NCP, NFP e PTF a heterogeneidade das variâncias é resultado do aumento da variância que ocorre devido ao aumento da média quando se agrupam as colheitas. Lúcio et al. (2010), estudando a variabilidade na fitomassa de frutos de tomate em estufas plásticas, também observaram que a variância sofre incrementos devido ao aumento da média quando agrupam-se as colheitas. Por serem valores médios, nas variáveis CMF, LMF, PMF e NFC não há este incremento, o que nos permite concluir que o agrupamento das colheitas reduz o valor da variância quando as colheitas são agrupadas em comparação com as colheitas individuais, acarretando na heterocedasticidade entre as colheitas.

A variabilidade existente entre as fileiras de cultivo para certos caracteres e entre as colheitas (individuais e agrupadas) dentro de uma mesma fileira de cultivo sugere a necessidade de dimensionar o tamanho da parcela para cada situação especifica. Independente da fileira de cultivo e do caractere estudado, o tamanho ótimo de parcela é menor quando as colheitas são agrupadas em ambas as estufas (Tabelas 3 e 4). O agrupamento de todas as colheitas reduz a variabilidade entre as plantas numa mesma fileira de cultivo, uma vez que minimiza a variabilidade decorrente da maturação desuniforme de frutos ou da presença de valores nulos no banco de dados, devido à ausência de frutos aptos para a colheita (Cargnelutti Filho et al., 2004; Lorentz et al., 2005; Lúcio et al. 2008, 2010).

O tamanho ótimo de parcela para estimar a fitomassa total de fruto (PTF) em tomate cereja é maior quando comparado ao tomate do tipo salada em ambiente protegido. Em trabalho realizado por Lúcio et al. (2010), os autores recomendam um tamanho de parcela de quatro 
plantas para estimar a fitomassa de frutos de tomate do tipo salada quando as colheitas são agrupadas. Já em relação a variável número de frutos por planta não há diferença no tamanho ótimo de parcela entre os tomates do tipo salada e cereja. Lúcio et al. (2012) recomendam a utilização de um tamanho de parcela de sete plantas na fileira de cultivo para o tomate do tipo salada, o mesmo que é necessário para a avaliação no tomate do tipo cereja quando as colheitas são agrupadas. Cabe ressaltar que não é possível fazer um paralelo entre os tipos de tomates para todas as variáveis devido a características distintas entre eles (como a presença de cachos no tomate do tipo cereja), ou pela ausência de trabalhos

Tabela 3. Tamanho ótimo de parcela (em número de plantas) para as variáveis comprimento médio de fruto (CMF), largura média de fruto (LMF), peso médio de fruto (PMF), número de cachos por planta $(\mathrm{NCP})$, número de frutos por cacho (NFC), número de frutos por planta (NFP) e peso total de fruto (PTF) no primeiro experimento sbest plot size (in number of plants) for the variables average length of fruit (CMF), average width of fruit (LMF), average weight of fruit (PMF), number of bunches per plant (NCP), number of fruits per bunch (NFC), number of fruits per plant (NFP) and total weight of fruit (PTF) in the first experiment . Santa Maria, UFSM, 2014.

\begin{tabular}{|c|c|c|c|c|c|c|c|c|c|c|c|c|}
\hline \multirow{3}{*}{ Fileiras } & \multicolumn{12}{|c|}{ Estufa pequena } \\
\hline & \multicolumn{6}{|c|}{ CMF } & \multicolumn{6}{|c|}{ LMF } \\
\hline & $\mathrm{Cl}^{1}$ & C2 & C3 & $\mathrm{C} 1+\mathrm{C} 2$ & $\mathrm{C} 2+\mathrm{C} 3$ & Todas & C1 & C2 & C3 & $\mathrm{C} 1+\mathrm{C} 2$ & $\mathrm{C} 2+\mathrm{C} 3$ & Todas \\
\hline $\mathrm{L}^{1}{ }^{1}$ & 5 & 7 & 5 & 1 & 4 & 1 & 5 & 7 & 5 & 2 & 4 & 2 \\
\hline L2 & 2 & 5 & 5 & 1 & 4 & 1 & 2 & 5 & 5 & 1 & 4 & 1 \\
\hline L3 & 5 & 4 & 4 & 2 & 4 & 2 & 5 & 4 & 4 & 2 & 4 & 2 \\
\hline L4 & 5 & 6 & 7 & 2 & 2 & 2 & 6 & 6 & 7 & 2 & 2 & 2 \\
\hline L5 & 2 & 2 & 4 & 2 & 2 & 2 & 2 & 2 & 4 & 2 & 2 & 2 \\
\hline L6 & 2 & 1 & 8 & 2 & 1 & 1 & 2 & 1 & 8 & 2 & 1 & 2 \\
\hline L7 & 4 & 6 & 7 & 4 & 2 & 2 & 4 & 6 & 7 & 4 & 2 & 2 \\
\hline \multirow[t]{2}{*}{ L8 } & 4 & 4 & 2 & 2 & 2 & 2 & 4 & 4 & 1 & 2 & 2 & 2 \\
\hline & \multicolumn{6}{|c|}{ PMF } & \multicolumn{6}{|c|}{ NCP } \\
\hline L1 & 6 & 7 & 5 & 3 & 4 & 3 & 8 & 9 & 8 & 7 & 8 & 6 \\
\hline L2 & 3 & 6 & 5 & 2 & 4 & 2 & 5 & 7 & 9 & 5 & 7 & 5 \\
\hline L3 & 6 & 5 & 4 & 4 & 4 & 3 & 7 & 8 & 9 & 6 & 7 & 6 \\
\hline L4 & 5 & 6 & 7 & 3 & 3 & 3 & 7 & 7 & 9 & 6 & 6 & 6 \\
\hline L5 & 4 & 3 & 4 & 3 & 2 & 3 & 6 & 7 & 8 & 4 & 6 & 4 \\
\hline L6 & 3 & 2 & 8 & 2 & 2 & 2 & 6 & 6 & 10 & 5 & 6 & 5 \\
\hline L7 & 5 & 6 & 7 & 5 & 3 & 3 & 7 & 7 & 10 & 6 & 7 & 6 \\
\hline \multirow[t]{2}{*}{ L8 } & 5 & 5 & 3 & 3 & 3 & 3 & 7 & 6 & 7 & 5 & 6 & 5 \\
\hline & \multicolumn{6}{|c|}{ NFC } & \multicolumn{6}{|c|}{ NFP } \\
\hline L1 & 8 & 8 & 10 & 5 & 7 & 6 & 9 & 9 & 10 & 7 & 8 & 6 \\
\hline L2 & 5 & 9 & 9 & 5 & 8 & 5 & 6 & 9 & 11 & 5 & 8 & 5 \\
\hline L3 & 7 & 7 & 8 & 4 & 7 & 4 & 8 & 9 & 11 & 6 & 8 & 6 \\
\hline L4 & 7 & 7 & 9 & 4 & 6 & 5 & 8 & 7 & 10 & 6 & 7 & 6 \\
\hline L5 & 5 & 7 & 9 & 5 & 7 & 4 & 6 & 8 & 11 & 5 & 7 & 5 \\
\hline L6 & 6 & 5 & 10 & 4 & 5 & 4 & 6 & 7 & 11 & 5 & 7 & 5 \\
\hline L7 & 6 & 8 & 9 & 5 & 6 & 4 & 7 & 8 & 10 & 6 & 7 & 6 \\
\hline L8 & 6 & 7 & 7 & 6 & 7 & 5 & 7 & 8 & 8 & 6 & 7 & 6 \\
\hline \multicolumn{13}{|c|}{ PTF } \\
\hline $\mathrm{L} 1$ & 8 & 9 & 10 & 7 & 9 & & & & & & & \\
\hline L2 & 5 & 9 & 11 & 5 & 9 & 5 & & & & & & \\
\hline L3 & 8 & 9 & 11 & 7 & 8 & 6 & & & & & & \\
\hline L4 & 7 & 8 & 10 & 6 & 7 & 6 & & & & & & \\
\hline L5 & 6 & 8 & 12 & 6 & 8 & 6 & & & & & & \\
\hline L6 & 6 & 7 & 11 & 5 & 7 & 5 & & & & & & \\
\hline L7 & 7 & 8 & 10 & 6 & 7 & 6 & & & & & & \\
\hline L8 & 7 & 8 & 9 & 7 & 8 & 7 & & & & & & \\
\hline
\end{tabular}

${ }^{1} \mathrm{C} 1=$ primeira colheita (first harvest); $\mathrm{C} 2=$ segunda colheita (second harvest); $\mathrm{C} 3=$ terceira colheita (third harvest); $\mathrm{C} 1+\mathrm{C} 2=$ primeira e segunda colheitas agrupadas (first and second harvests, grouped); $\mathrm{C} 2+\mathrm{C} 3=$ segunda e terceira colheitas agrupadas (second and third harvests, grouped); Todas= todas as colheitas agrupadas (all harvests, grouped). L1 a L8= fileiras 1 a 8 (rows 1 to 8). 
que estimem o tamanho ótimo de parcela para determinadas variáveis em outros tipos de tomate.

Para todas as variáveis estudadas observou-se que a autocorrelação espa- cial de primeira ordem não tem influencia na estimativa do tamanho ótimo de parcela, e que a média correlacionou-se negativamente com o tamanho ótimo de parcela na maioria das variáveis analisa- das. Para as variáveis CMF, LMF, PMF e NFC a variância e o tamanho ótimo de parcela correlacionam-se de forma positiva, ou seja, as maiores variâncias estão associadas a maiores estimativas

Tabela 4. Tamanho ótimo de parcela (em número de plantas) para as variáveis comprimento médio de fruto (CMF), largura média de fruto (LMF), peso médio de fruto (PMF), número de cachos por planta (NCP), número de frutos por cacho (NFC), número de frutos por planta (NFP) e peso total de fruto (PTF) no segundo experimento best plot size (in number of plants) for the variables average length of fruit (CMF), average width of fruit (LMF), average weight of fruit (PMF), number of bunches per plant (NCP), number of fruits per bunch (NFC), number of fruits per plant (NFP) and total weight of fruit (PTF) in the second experiment\}. Santa Maria, UFSM, 2014.

\begin{tabular}{|c|c|c|c|c|c|c|c|c|c|c|c|c|}
\hline \multirow{3}{*}{ Fileiras } & \multicolumn{12}{|c|}{ Estufa grande } \\
\hline & \multicolumn{6}{|c|}{ CMF } & \multicolumn{6}{|c|}{ LMF } \\
\hline & $\mathrm{C1}^{1}$ & $\mathrm{C2}$ & C3 & $\mathrm{C} 1+\mathrm{C} 2$ & $\mathrm{C} 2+\mathrm{C} 3$ & Todas & C1 & $\mathrm{C} 2$ & C3 & $\mathrm{C} 1+\mathrm{C} 2$ & $\mathrm{C} 2+\mathrm{C} 3$ & Todas \\
\hline $\mathrm{L} 1$ & 6 & 5 & 7 & 5 & 2 & 2 & 6 & 5 & 7 & 5 & 2 & 2 \\
\hline L2 & 6 & 5 & 5 & 2 & 4 & 2 & 6 & 5 & 5 & 2 & 4 & 2 \\
\hline L3 & 9 & 5 & 6 & 4 & 4 & 4 & 9 & 5 & 6 & 4 & 4 & 4 \\
\hline L4 & 8 & 5 & 6 & 4 & 4 & 4 & 8 & 5 & 6 & 4 & 4 & 4 \\
\hline L5 & 6 & 6 & 5 & 2 & 2 & 1 & 6 & 6 & 5 & 2 & 2 & 1 \\
\hline L6 & 9 & 5 & 8 & 5 & 5 & 5 & 9 & 5 & 8 & 5 & 5 & 5 \\
\hline L7 & 6 & 6 & 6 & 1 & 4 & 1 & 6 & 6 & 6 & 1 & 4 & 1 \\
\hline \multirow[t]{2}{*}{ L8 } & 9 & 6 & 7 & 5 & 5 & 5 & 10 & 6 & 7 & 5 & 5 & 5 \\
\hline & \multicolumn{6}{|c|}{ PMF } & \multicolumn{6}{|c|}{ NCP } \\
\hline $\mathrm{L} 1$ & 6 & 5 & 7 & 5 & 7 & 3 & 9 & 7 & 10 & 7 & 6 & 6 \\
\hline L2 & 6 & 5 & 5 & 3 & 9 & 3 & 9 & 7 & 9 & 6 & 7 & 6 \\
\hline L3 & 9 & 6 & 6 & 4 & 10 & 4 & 11 & 7 & 8 & 7 & 6 & 6 \\
\hline L4 & 8 & 5 & 6 & 4 & 9 & 4 & 9 & 6 & 9 & 6 & 6 & 6 \\
\hline L5 & 6 & 6 & 5 & 3 & 7 & 3 & 8 & 9 & 7 & 8 & 7 & 6 \\
\hline L6 & 10 & 6 & 8 & 5 & 11 & 5 & 11 & 7 & 10 & 6 & 7 & 7 \\
\hline L7 & 7 & 7 & 6 & 3 & 10 & 3 & 8 & 9 & 8 & 7 & 7 & 6 \\
\hline \multirow[t]{2}{*}{ L8 } & 10 & 6 & 7 & 6 & 11 & 5 & 11 & 8 & 9 & 7 & 7 & 7 \\
\hline & \multicolumn{6}{|c|}{ NFC } & \multicolumn{6}{|c|}{ NFP } \\
\hline L1 & 8 & 6 & 9 & 6 & 5 & 5 & 10 & 7 & 11 & 7 & 6 & 6 \\
\hline L2 & 9 & 6 & 7 & 5 & 6 & 5 & 9 & 8 & 9 & 6 & 7 & 6 \\
\hline L3 & 11 & 7 & 9 & 6 & 6 & 5 & 11 & 8 & 10 & 8 & 7 & 7 \\
\hline L4 & 11 & 6 & 7 & 6 & 5 & 5 & 11 & 7 & 10 & 7 & 7 & 6 \\
\hline L5 & 8 & 7 & 8 & 4 & 5 & 4 & 9 & 8 & 9 & 7 & 7 & 7 \\
\hline L6 & 11 & 7 & 9 & 6 & 6 & 6 & 12 & 7 & 11 & 6 & 7 & 6 \\
\hline L7 & 9 & 8 & 9 & 4 & 5 & 4 & 9 & 9 & 8 & 8 & 7 & 7 \\
\hline \multirow[t]{2}{*}{ L8 } & 11 & 7 & 9 & 6 & 6 & 5 & 13 & 8 & 10 & 8 & 7 & 7 \\
\hline & \multicolumn{6}{|c|}{ PTF } & & & & & & \\
\hline L1 & 10 & 7 & 11 & 7 & 6 & 6 & & & & & & \\
\hline L2 & 10 & 8 & 9 & 6 & 7 & 6 & & & & & & \\
\hline L3 & 12 & 8 & 10 & 8 & 7 & 7 & & & & & & \\
\hline L4 & 11 & 7 & 11 & 7 & 7 & 7 & & & & & & \\
\hline L5 & 9 & 8 & 10 & 7 & 7 & 7 & & & & & & \\
\hline L6 & 12 & 7 & 11 & 6 & 7 & 6 & & & & & & \\
\hline L7 & 10 & 9 & 9 & 8 & 7 & 7 & & & & & & \\
\hline L8 & 13 & 8 & 10 & 7 & 7 & 7 & & & & & & \\
\hline
\end{tabular}

${ }^{1} \mathrm{C} 1=$ primeira colheita; $\mathrm{C} 2=$ segunda colheita; $\mathrm{C} 3=$ terceira colheita; $\mathrm{C} 1+\mathrm{C} 2=$ primeira e segunda colheitas agrupadas; $\mathrm{C} 2+\mathrm{C} 3=$ segunda $\mathrm{e}$ terceira colheitas agrupadas; Todas $=$ todas as colheitas agrupadas $(\mathrm{C} 1=$ first harvest; $\mathrm{C} 2=$ second harvest; $\mathrm{C} 3=$ third harvest; $\mathrm{C} 1+\mathrm{C} 2=$ first and second harvests, grouped; $\mathrm{C} 2+\mathrm{C} 3=$ second and third harvests, grouped; All = all harvests, grouped). 
Tabela 5. Coeficientes de correlação de Pearson entre as estatísticas tamanho de parcela $(\hat{X} o)$, média $(\bar{X})$, autocorrelação espacial de primeira ordem $(\hat{\rho})$ e variância $\left(\mathrm{s}^{2}\right)$ para as variáveis comprimento médio de fruto $(\mathrm{CMF})$, largura média de fruto (LMF), peso médio de fruto $(\mathrm{PMF})$, número de cachos por planta (NCP), número de frutos por cacho (NFC), número de frutos por planta (NFP) e peso total de fruto (PTF) \{Pearson's correlation coefficients among the statistics of plot size $(\hat{X} o)$, mean $(\bar{X})$, spatial autocorrelation of the first order $(\hat{p})$ and variance $\left(\mathrm{s}^{2}\right)$ for the variables average length of fruit $(\mathrm{CMF})$, average width of fruit (LMF), average weight of fruit (PMF), number of bunches per plant (NCP), number of fruits per bunch (NFC), number of fruits per plant (NFP) and total weight of fruit (PTF) . Santa Maria, UFSM, 2014.

\begin{tabular}{|c|c|c|c|c|c|c|c|c|c|}
\hline & \multicolumn{4}{|c|}{ CMF } & \multicolumn{5}{|c|}{ LMF } \\
\hline & $\hat{X}_{o}$ & $\bar{x}$ & $\hat{\rho}$ & $\mathbf{s}^{2}$ & & $\hat{X}_{o}$ & $\bar{x}$ & $\hat{\rho}$ & $\mathbf{s}^{2}$ \\
\hline$\hat{X}_{o}$ & 1 & $-0,72^{*}$ & $-0,22$ & $0,94^{*}$ & $\hat{X}_{o}$ & 1 & $-0,69^{*}$ & $-0,10$ & $0,94^{*}$ \\
\hline $\bar{x}$ & & 1 & 0,05 & $-0,67 *$ & $\bar{x}$ & & 1 & $-0,06$ & $-0,62 *$ \\
\hline$\hat{\rho}$ & & & 1 & $-0,12^{*}$ & $\hat{\rho}$ & & & 1 & $-0,04$ \\
\hline \multirow[t]{2}{*}{$\mathrm{S}^{2}$} & & & & 1 & $\mathrm{~s}^{2}$ & & & & 1 \\
\hline & \multicolumn{4}{|c|}{ PMF } & \multicolumn{5}{|c|}{ NCP } \\
\hline$\hat{X}_{o}$ & 1 & $-0,18$ & $-0,04$ & $0,60 *$ & $\hat{X}_{O}$ & 1 & $-0,77^{*}$ & 0,05 & $-0,34^{*}$ \\
\hline $\bar{x}$ & & 1 & 0,04 & 0,11 & $\bar{x}$ & & 1 & $-0,15$ & $0,81^{*}$ \\
\hline$\hat{\rho}$ & & & 1 & $-0,08$ & $\hat{\rho}$ & & & 1 & $-0,17$ \\
\hline \multirow[t]{2}{*}{$\mathrm{s}^{2}$} & & & & 1 & $\mathrm{~s}^{2}$ & & & & 1 \\
\hline & \multicolumn{4}{|c|}{ NFC } & \multicolumn{5}{|c|}{ NFP } \\
\hline$\hat{X}_{o}$ & 1 & $-0,60 *$ & $-0,05$ & $0,59 *$ & $\hat{X}_{o}$ & 1 & $-0,86^{*}$ & $-0,05$ & $-0,61 *$ \\
\hline $\bar{x}$ & & 1 & $-0,02$ & $0,24^{*}$ & $\bar{x}$ & & 1 & $-0,10$ & $0,87^{*}$ \\
\hline$\hat{\rho}$ & & & 1 & $-0,07$ & $\hat{\rho}$ & & & 1 & $-0,16$ \\
\hline $\mathrm{s}^{2}$ & & & & 1 & $\mathrm{~s}^{2}$ & & & & 1 \\
\hline \multicolumn{10}{|c|}{ PTF } \\
\hline$\hat{X}_{o}$ & 1 & $-0,87^{*}$ & $-0,09$ & $-0,62 *$ & & & & & \\
\hline $\bar{x}$ & & 1 & $-0,07$ & $0,88^{*}$ & & & & & \\
\hline$\hat{\rho}$ & & & 1 & $-0,13$ & & & & & \\
\hline $\mathrm{s}^{2}$ & & & & 1 & & & & & \\
\hline
\end{tabular}

*Efeito significativo pelo teste $\mathrm{t}$, a $5 \%$ de probabilidade, com 94 graus de liberdade (significant effect at 5\%, with 94 degrees of freedom).

lineamento experimental adequado são formas de diminuir o erro experimental. No caso de experimentos com tomate cereja, devido à variabilidade existente entre as fileiras de cultivo para algumas variáveis, recomenda-se a adoção do delineamento blocos ao acaso (Lorentz et al., 2005).

Como forma de redução da variabilidade e melhor aproveitamento da área, é recomendado que para avaliar as variáveis CMF, LMF, PMF, NCP, NFC, NFP e PTF as colheitas sejam agrupadas e que a parcela seja composta de no mínimo sete plantas de tomate cereja, independentemente da fileira de cultivo, sendo esta a primeira recomendação para a cultura, e para um elevado número de variáveis. Na impossibilidade de serem colhidos todos os frutos, recomenda-se a adoção dos maiores tamanhos de parcela estimados neste trabalho, ficando a decisão a critério do pesquisador. Por fim, incentiva-se a realização de ensaios de repetibilidade que determinem o número mínimo de colheitas necessárias para a comparação de tratamentos e genótipos de tomate tipo cereja.

Conclui-se, portanto, que existe heterocedasticidade entre as fileiras de cultivo para pelo menos uma das variáveis estudadas, independente do agrupamento ou não de colheitas. Há também heterocedasticidade entre as colheitas individuais e agrupadas para todas as variáveis estudadas para uma mesma fileira de cultivo. O agrupamento de colheitas reduz a variabilidade e, consequentemente, a estimativa do tamanho da parcela. O tamanho ótimo de parcela para avaliar o comprimento médio de fruto, a largura média de fruto, o peso médio de fruto, o número de cachos, o número de frutos por cacho, o número de frutos e o peso total de frutos é de sete plantas quando todas as colheitas são agrupadas.

\section{REFERÊNCIAS}

AZEVEDO, VF; ABBOUD, AD; CARMO, MGF. 2010. Row spacing and pruning regimes on organically grown cherry tomato. Horticultura Brasileira 28: 389-394.

CARGNELUtTi FILHO, A; RADIN, B; MATZENAUER, R; STORCK, L. 2004. de tamanho ótimo de parcela e, consequentemente, a maior variabilidade. Porém, para as variáveis NCP, NFP e PTF, a média e a variância correlacionam-se de forma positiva, comprovando que com o agrupamento de colheitas há incremento nos valores da variância. Por isso, para estas variáveis, a variância e as estimativas do tamanho ótimo de parcela correlacionam-se de forma negativa, ou seja, quanto maior a variância menor o tamanho de parcela (Tabela 5). Ressalta-se, deste modo, que os valores mais elevados da variância não estão
Os resultados apresentados ressaltam a existência de variabilidade em experimentos com tomate cereja em ambiente protegido, e que esta deve ser controlada. Conforme Storck et al. (2006), o dimensionamento quanto ao tamanho de parcela e a utilização de de-

relacionados com a maior variabilidade entre as plantas no caso destas variáveis uma vez que, quando as colheitas adas, a variabilidade é redu , consequentemente, a estimativa do tamanho ótimo da parcela é menor (Tabelas 3 e 4).

Hortic. bras., v. 34, n. 2, abr. - jun. 2016 
Número de colheitas e comparação de genótipos de tomateiro cultivados em estufa de plástico. Pesquisa Agropecuária Brasileira 39: 953-959.

CARPES, RH; LÚCIO, ADC; LOPES, SJ; BENZ, V; HAESBAERT, F; SANTOS, D. 2010. Variabilidade produtiva e agrupamentos de colheitas de abobrinha italiana cultivada em ambiente protegido. Ciência Rural 40: 294-301.

CERMEÑO, ZS. 1990. Estufas - instalações e manejo. Lisboa: Litexa. 355p.

EMBRAPA. 2006. Sistema brasileiro de classificação de solos. Rio de Janeiro: Centro Nacional de Pesquisa de Solos. 306p.

FEIJÓ, S; OLIVEIRA, SJR; STORCK, L; LÚCIO, ADC; DAMO, HP; MARTINI, LFD. 2005. Repetibilidade da produção de frutos de abobrinha italiana. Revista Brasileira de Agrociência 11: 39-43.

FEIJÓ, S; STORCK, L; LÚCIO, ADC; LOPES, SJ; GRACIA, DC; CARPES, RH. 2008. Heterogeneity index of zucchini yield on a protected environment and experimental planning. Horticultura Brasileira 26: 35-39.

GUSMÃO, MTA; GUSMÃO, SAL; ARAÚJO, JAC. 2006. Produtividade de tomate tipo cereja cultivado em ambiente protegido e em diferentes substratos. Horticultura Brasileira 24: 431-436.

GUSMÃO, SAL; PÁDUA, JG; GUSMÃO, MTA; BRAZ, LT. 2000. Efeito da densidade de plantio e forma de tutoramento na produção de tomateiro tipo "cereja" em Jaboticabal-SP. Horticultura Brasileira 18: 572-573.

IBGE. 2014. Levantamento sistemático da produção agrícola: pesquisa mensal de previsão e acompanhamento das safras agrícolas no ano civil. Rio de Janeiro: IBGE. $85 \mathrm{p}$.

LORENTZ, LH; LÚCIO, ADC; BOLIGON, AA; LOPES, SJ; STORCK, L. 2005. Variabilidade da produção de frutos de pimentão em estufa plástica. Ciência Rural 35: 316-323.

LORENTZ, LH; LÚCIO, ADC; STORCK, L; LOPES, SJ; BOLIGON, AA; CARPES, RH. 2004. Variação temporal do tamanho de amostra para experimentos em estufa plástica. Ciência Rural 34: 1043-1049.

LÚCIO, ADC; CARPES, RH; STORCK, L; LOPES, SJ; LORENTZ, LH; PALUDO, AL. 2008. Variância e média da massa de frutos de abobrinha-italiana em múltiplas colheitas. Horticultura Brasileira 26: 335-341.

LÚCIO, ADC; CARPES, RH; STORCK, L; ZANARDO, B; TOEBE, M; PUHL, J; SANTOS, JRA. 2010. Agrupamento de colheitas de tomate e estimativas do tamanho de parcela em cultivo protegido. Horticultura Brasileira 28: 190-196.

LÚCIO, ADC; HAESBAERT, FM; SANTOS, D; SCHWERTNER, DV; BRUNES, RR. 2012. Tamanhos de amostra e de parcela para variáveis de crescimento e produtivas de tomateiro. Horticultura Brasileira 30: 660-668.

LÚCIO, ADC; LORENTZ, LH; BOLIGON, AA; LOPES, SJ; STORCK, L; CARPES, RH. 2006. Variação temporal da produção de pimentão influenciada pela posição e características morfológicas das plantas em ambiente protegido. Horticultura Brasileira 24: 31-35.

LÚCIO, ADC; MELLO, RM; STORCK, L; CARPES, RH; BOLIGON, AA; ZANARDO, B. 2004. Estimativa de parâmetros para o planejamento de experimentos com a cultura do pimentão em área restrita. Horticultura Brasileira 22: 766-770.

LÚCIO, ADC; SOUZA, MF; HELDWEIN, AB; LIEBERKNECHT, D; CARPES, RH; CARVALHO, MP. 2003. Tamanho da amostra e método de amostragem para avaliação de características do pimentão em estufa plástica. Horticultura Brasileira 21: 80-184.

PARANAÍBA, PF; FERREIRA, DF; MORAIS, AR. 2009. Tamanho ótimo de parcelas experimentais: proposição de métodos de estimação. Revista Brasileira de Biometria 27: 255-268.

R DEVELOPMENT CORE TEAM. 2014. R: A language and environment for statistical computing: R Foundation for Statistical Computing version 2.15.1 (software). Austria.

ROCHA, MQ; PEIL, RMN; COGO, CM. 2010. Rendimento do tomate cereja em função do cacho floral e da concentração de nutrientes em hidroponia. Horticultura Brasileira 28: 466-471.

ROLAS. 2004. Recomendações de adubações e de calagem para os estados do Rio Grande do Sul e Santa Catarina. Passo Fundo: SBCS, Núcleo Regional Sul. 224p.

SANTOS, D; HAESBAERT, FM; LÚCIO, ADC; STORCK, L; CARGNELUTTI FILHO, A. 2012. Tamanho ótimo de parcela para a cultura do feijão-vagem. Revista Ciência Agronômica 43: 119-128.

STEEL, RGD; TORRIE, JH; DICKEY, DA. 1997. Principles and procedures of statistics: a biometrical approach. 3ed. New York: McGraw-Hill. 666p.

STORCK, L; GARCIA, DC; LOPES, SJ; ESTEFANEL, V. 2006. Experimentação vegetal. Santa Maria: UFSM. 198p. 